\title{
On Key Storage in Secure Networks
}

\author{
Martin Dyer* \\ School of Computer Studies, University of Leeds, \\ Leeds, England \\ Trevor Fenner \\ Department of Computer Science, Birkbeck College, \\ University of London, London, England \\ Alan Frieze** \\ Department of Mathematics, Carnegie-Mellon University, \\ Pittsburgh, U.S.A. \\ Andrew Thomason \\ Department of Pure Mathematics and Mathematical Statistics, \\ University of Cambridge, Cambridge, England \\ Communicated by Ernest F. Brickell \\ Received 21 August 1992 and revised 8 November 1994

\begin{abstract}
We consider systems where the keys for encrypting messages are derived from the pairwise intersections of sets of private keys issued to the users. We give improved bounds on the storage requirements of systems of this type for secure communication in a large network.
\end{abstract}

Key words. Keys, Secure networks, Probabilistic construction.

\section{Introduction}

The problem of secure communication in a network with multiple users has been considered by Blom [2], [3], Mitchell and Piper [8], Li Gong and Wheeler [7], and other authors. These papers consider a solution in which a keydistribution centre (KDC) must, in principle, issue a unique cryptographic key to each of the pairs of users in a network whenever they choose to begin communication. Suppose $N=\{1,2, \ldots, n\}$ is the set of users. Then, since there are $\left(\begin{array}{l}n \\ 2\end{array}\right)$ possible pairs, if $n$ is large a direct implementation will involve the

\footnotetext{
*Supported by NATO Grant RG0088/89.

${ }^{* *}$ Supported by NSF Grant CCR-8900112 and NATO Grant RG0088/89.
} 
storage of about $\frac{1}{2} n^{2}$ keys. In modern computer networks it is, in fact, very likely that $n$ will be rather large, and current trends seem likely to increase the number of users attached to such networks.

In [8] Mitchell and Piper, inspired by a proposal of Blom [2], [3], considered a scheme in which the KDC stores some global set $K$ of keys $(|K|=k)$ and issues to each user $i(i=1,2, \ldots, n)$ a subset $S_{i} \subseteq K$ of these keys. We assume these keys are numbered arbitrarily $1,2, \ldots, k$. There is a directory, which need not be secure, which lists the numbers of the keys held by each user. Now, if user $i$ wishes to communicate with user $j$, the key to be used is constructed from the set of keys contained in $S_{i} \cap S_{i}$. (Information about which numbered keys uses $i$ and $j$ hold can be exchanged without secure communication.) We insist that for no other user, $r$, is it true that $S_{i} \cap S_{j} \subseteq S_{r}$. The required keys are then clearly available to both users but to no other single user. These keys can then be transformed, possibly via some one-way function, into the key to be used to encrypt all communication between users $i$ and $j$. Mitchell and Piper discuss various schemes, some of which require only $O(n)$ keys in total, as opposed to the $\Omega\left(n^{2}\right)$ required by a direct implementation. Li Gong and Wheeler [7] give a different scheme, requiring $O(n)$ keys in total, with each user required only to hold $O(\sqrt{n})$ keys.

We call schemes of the type discussed above set intersection schemes. In this paper, in Section 2, we demonstrate the existence of set intersection schemes requiring only $O(\log n)$ keys in total, and hence a fortiori each user has to hold only $O(\log n)$ keys. We show that this is optimal to within constant factors. Constant factors are, of course, very important in applications, but we also show that they are of quite reasonable size in our scheme. Our construction is an application of the probabilistic method in combinatorics [9]. This is a simple yet robust method for obtaining combinatorial constructions, which perhaps deserves to be more widely known in the cryptography community. In Section 3 we give some experimental evaluations of our proposals to demonstrate their practical feasibility. To counter possible objections to the use of random methods, we further show in Section 4 that, if necessary, the construction can be "derandomized" by the method of conditional probabilities [9].

A known difficulty with set intersection schemes [8], [7] is the problem of collusion. If all members of some group $W \subseteq N(|W|=w)$ choose to disclose their keys to one of their number, this user may then possess the subset of keys which two others, not in the group, are using to communicate, i.e., for some $i, j$ and $W \subseteq N$ we have

$$
S_{i} \cap S_{j} \subseteq \bigcup_{r \in W^{\prime}} S_{r} .
$$

This clearly compromises the network for secure communication between users $i$ and $j$. We examine this problem in Section 5 and give upper and lower bounds on the number of keys required to ensure network security against groups of at most $w$ colluders, for any given $w$. In Section 6 we consider a generalization of the problem to communication between groups of (more than two) users, and in Section 7 we make some concluding remarks. 


\section{A Space-Efficient Key-Storage System}

We use $\lg n$ for $\log _{2} n$. Our first simple result also appears, in a different form, in $[8]$.

Lemma 1. Any set intersection scheme requires each user to hold at least $\lg n$ keys, and at least $2 \lg n$ keys in total $(n \geq 4)$.

Proof. Any user who has less than $\lg n$ keys can form less than $n-1$ distinct nonempty subsets from their keys. Thus they cannot have a different intersection with each of the other $n-1$ users. There are $\left(\begin{array}{l}n \\ 2\end{array}\right)$ pairs of users, and their key intersections must form a set antichain. It follows that we must have

$$
\left(\begin{array}{c}
k \\
\lfloor k / 2\rfloor
\end{array}\right) \geq\left(\begin{array}{l}
n \\
2
\end{array}\right)
$$

This implies $k \geq 2 \lg n$ for all $n \geq 4$.

We thus have a very straightforward demonstration that the number of keys needed is at least of the order of $\log n$. Using a somewhat more delicate argument than the crude proof of Lemma 1, Erdős et al. [5] showed that at least $3.1 \mathrm{lg} n$ keys are needed. In fact, set intersection schemes for $n$ users using only $O(\log n)$ keys do exist. We show how probabilistic arguments can be used to find such schemes. However, we note here that, again by an argument similiar to but more delicate than that which we employ, Erdös et al. [5] have shown the existence of schemes with only $5.6 \lg n$ keys. Our aim is to show how schemes which are not much bigger can be contructed easily.

We now show:

Theorem 1. A set intersection scheme requiring only $\lceil 13 \lg n\rceil$ keys in total exists.

Proof. Suppose the sets are generated randomly in some way. Let $X_{i s}$ be the random variable which is the indicator of the event $s \in S_{i}$. A "bad triple" is a triple $(i, j, r)$ such that $S_{i} \cap S_{j} \subseteq S_{r}$. Thus the expected number of bad triples is

$$
\mathbf{E}\left(\sum_{r=1}^{n} \sum_{\substack{i=1 \\ i \neq r}}^{n} \sum_{\substack{j=1 \\ j \neq r}}^{i-1} \prod_{\substack{s=1 \\ j \neq r}}^{k}\left(1-X_{i s} X_{j s}\left(1-X_{r s}\right)\right)\right) .
$$

This is an upper bound on the probability of the existence of any bad triple. Thus, if the $X_{i s}$ are independent Bernoulli variables with $\operatorname{Pr}\left(X_{i s}=1\right)=p$, then

$$
\operatorname{Pr}(\text { any bad triple }) \leq n\left(\frac{n}{2}\right)\left(1-p^{2}(1-p)\right)^{k} \leq \frac{1}{2} n^{3}\left(1-p^{2}(1-p)\right)^{k} .
$$


This is minimized by choosing $p=\frac{2}{3}$. Then if $k=13 \lg n$, it is easy to check that

$$
\operatorname{Pr}(\text { any bad triple }) \leq \frac{1}{2} n^{3}\left(\frac{23}{27}\right)^{13 \lg n} \leq \frac{1}{2} n^{-0.007}<\frac{1}{2} .
$$

Thus if we generate the sets randomly in this manner, we have a probability of more than one-half that the scheme we generate will be "good."

Observe that we may check that the generated scheme has the required properties. This can be done by an $O\left(n^{3} k\right)$ computation, by checking all triples to ensure that they are not bad. This is feasible for small $n$, and could easily be implemented in parallel. In fact, this checking problem is easily seen to be in the class NC of efficiently parallelizable problems. It may be observed that the whole scheme could be generated and checked by a randomized parallel computation. Thus generating and checking is in the parallel complexity class RNC.

\section{Practical Schemes}

From a practical point of view, the moral of the previous section is that set intersection schemes are best generated at random. For example, it is instructive to compare the bound of Theorem 1 with those of [7]. The matrix schemes of [7] require at least $n$ keys. Now $13 \lg n<n$ for all $n \geq 83$. If $n=1000$, say, we have $[13 \lg n]=130$, less than one-seventh of the key requirement. The total number of keys stored by all users is at least $3 n^{3 / 2}$ for the method of [7], whereas ours is approximately $\frac{26}{3} n \lg n$. This is smaller for $n \geq 800$, say. Lest this should appear a disadvantage of our method, it should be observed that these comparisons are not entirely fair to our scheme, since those of [7] are only proven to be free of bad triples if the total number of keys stored is at least $4 n^{3 / 2}$-in which case our method gives a smaller total for $n \geq 350$, say. Also, we have based our bound for $k$ on estimates of probabilities which may be pessimistic for small $n$.

In practice, we can in fact do rather better than Theorem 1 would imply. We chose a very simple random model to make the theoretical analysis easy; in practice, it is more efficient to choose other models. In particular, rather than select, for each user, a random subset of the $k$ keys by selecting each with probability $p$, it is better to give each user a random subset of size $t$, for some fixed $t$. The best value of $t$ to choose would seem to be that which minimizes the probability that a randomly chosen triple is "bad", in the sense of the proof of Theorem 1 . This probability is

$$
p_{k, t}=\sum_{u=0}^{t}\left(\begin{array}{l}
t \\
u
\end{array}\right)\left(\begin{array}{c}
k-t \\
t-u
\end{array}\right)\left(\begin{array}{c}
k-u \\
t-u
\end{array}\right)\left(\begin{array}{l}
k \\
t
\end{array}\right)^{-2},
$$

$u$ being the size of the intersection $S_{i} \cap S_{j}$. Usually the minimum value of $p_{k, t}$ is less than $\left(\frac{23}{27}\right)^{k}$, and can be substituted for $\left(\frac{23}{27}\right)^{k}$ in (3) to demonstrate the 
Table 1. The existence of set intersection schemes.

\begin{tabular}{ccccc}
\hline & \multicolumn{4}{c}{ Number of keys required } \\
\cline { 2 - 5 } Users & Thm. 1 & $t$-sets & Risk $10^{-10}$ & Risk $10^{-20}$ \\
\hline 50 & 73 & 48 & & \\
75 & 80 & 53 & & \\
100 & 86 & 56 & & \\
200 & 99 & 64 & 152 & \\
500 & 116 & 75 & 163 & 254 \\
$10^{3}$ & 129 & 83 & 171 & 262 \\
$10^{4}$ & 172 & 111 & 199 & 290 \\
$10^{5}$ & 215 & 138 & 226 & 317 \\
$10^{6}$ & 258 & 165 & 253 & 344 \\
$20^{8}$ & 344 & 220 & 308 & 399 \\
\hline
\end{tabular}

existence of a scheme of $n$ users with fewer keys than required by Theorem 1 . For small values of $n$ the gain is striking. For 100 users, Theorem 1 (or its proof) shows the existence of a scheme with 86 keys, the number of keys rising to 116 for 500 users, 129 for 1000 users, and 258 for a million users. This is already dramatically less than [7], but the method of choosing $t$-sets allows these figures to be reduced to 56 keys, 75 keys, 83 keys, and 165 keys, respectively. Table 1 gives further data; in this table the column labelled "Thm. 1" gives the upper bound on the number of keys required by the proof of Theorem 1, and the column labelled " $t$-sets" gives the improved upper bound given by the method of choosing $t$-sets.

The method is genuinely practical. For 500 users the schemes generated can be tested on a SUN SPARCstation 1 in 2 minutes, rising to 16 minutes for 1000 users, with an eightfold increase in time for every doubling of the number of users. For much larger numbers of users, each user could be asked to check his own keys, thus performing the checking computation in a "distributed" manner; with 100,000 users a user can check his own keys in about 5 hours (sequential) time on the SPARC. (Of course a user need only check his own keys for secure communication with those other users with whom he anticipates communicating, and he can do this in less than $\frac{1}{2}$ second per user.)

If an astronomically large number of users is envisaged, a value of $k$ can be chosen to ensure the probability of an incorrect scheme being generated is less than, say, $10^{-20}$. For 100 million users, $k=399$ would suffice. This is verified by checking that, with $n=10^{8}$ and $k=399, \frac{1}{2} n^{3} p_{k, t}<10^{-20}$ holds. Values for $k$ ensuring that a randomly chosen collection of $t$-sets will give a set intersection scheme for $n$ users with probability at least $1-10^{-20}$ are given in the "risk $10^{-20}$ " column of Table 1; the "risk $10^{-10}$ " column has an analogous meaning.

An efficient way to generate schemes in practice is to select a suitable value of $k$, compute the best value of $t$ as above, and then randomly select, one by one, subsets of size $t$, stopping when one of them forms a bad triple with two previous choices. In this way quite large schemes can be found with relatively few keys. Sometimes a bad choice is made early on; the method can be made less susceptible to such bad luck by allowing another random choice after a 
Table 2. Set intersection schemes found by the "retry" method.

\begin{tabular}{ccrrr}
\hline & & \multicolumn{3}{c}{ Number of users } \\
\cline { 3 - 5 } Keys & Fails & Min & Mean & Max \\
\hline 40 & 0 & 14 & 33 & 69 \\
40 & 1 & 49 & 68 & 89 \\
40 & 2 & 84 & 97 & 111 \\
40 & 5 & 106 & 137 & 153 \\
50 & 0 & 41 & 84 & 155 \\
50 & 1 & 115 & 168 & 237 \\
50 & 2 & 190 & 248 & 303 \\
50 & 5 & 314 & 414 & 489 \\
60 & 0 & 92 & 141 & 201 \\
60 & 1 & 201 & 470 & 637 \\
60 & 2 & 307 & 711 & 962 \\
60 & 5 & 1111 & 1260 & 1387 \\
70 & 0 & 186 & 392 & 503 \\
70 & 1 & 663 & 1292 & 1732 \\
70 & 2 & 1481 & 2141 & 2571 \\
70 & 5 & 3236 & 3787 & 4408 \\
\hline
\end{tabular}

failed choice, and another choice again if necessary, up to a specified limit on the number of successive failures before stopping. This method, which we call the "retry" method, has been tried in practice and the results are summarized in Table 2. For each value of $k$ (in the "keys" column), and number of failures allowed (in the "fails" column), 10 attempts were made to generate schemes by this method. The table shows the minimum, mean, and maximum number of users in the final schemes. Note that the number of keys needed for a given number of users is dramatically less even than that given by Table 1; for instance a scheme for 3000 users with only 70 keys can be found in just minutes of computer time.

\section{Derandomization}

While, in practice, we believe that an efficient randomized scheme is always practically acceptable (see Section 7 below), from a theoretical point of view we might wish to dispense with the requirement for randomization. This can easily be achieved using the method of conditional probabilities [9].

Theorem 2. The set intersection scheme of Theorem 1 can be generated by an $O\left(n^{3} k\right)$-time deterministic algorithm using $O\left(n^{3}\right)$ storage.

Proof. Suppose we construct the sets by fixing one $X_{i s}$ at a time in major order $i=1,2, \ldots, n$ and minor order $s=1,2, \ldots, k$. We ensure that initially the probability of a bad triple, as given by (2), is less than 1 . When we come to $X_{i s}$ the "previous" $X$ 's will have been fixed (at 0 or 1) in (1). The following 
"unknown" $X$ 's will still be Bernoulli random variables with probability $p\left(=\frac{2}{3}\right.$ here). Assume the value of (1) at this stage is $E<1$. We now, try in turn, both cases for $X_{i s}=0,1$ in (1) and evaluate the expected value. The reader may check that this can be accomplished by an $O\left(n^{2}\right)$ computation of $E$ and the $O\left(n^{3}\right)$ products of which it is the sum are stored and updated whenever an $X_{i s}$ "changes" from $p$ to 0 or 1 . Let the two expected values calculated in this way be $E_{0}, E_{1}$. Then clearly

$$
E=(1-p) E_{0}+p E_{1} .
$$

Thus if $E<1$, then either $E_{0}<1$ or $E_{1}<1$. We fix $X_{i s}=0$ if $E_{0}<1, X_{i s}=1$ if $E_{1}<1$, and proceed to the next variable. (If both $E_{0}<1$ and $E_{1}<1$, we may choose arbitrarily.) Obviously we must terminate with $E=0$, and hence we will have constructed the desired scheme. The algorithm takes $n k \times O\left(n^{2}\right)=O\left(n^{3} k\right)$ time.

It may be observed that this is only of the same complexity as checking the randomized construction. However, there is an important difference from a practical point of view. The derandomized algorithm does not appear to be efficiently parallelizable.

\section{The Problem of Collusion}

We consider here the collusion problem mentioned in Section 1. Let us first establish a lower bound on $k$ in this context.

Theorem 3. Any set intersection scheme which is secure against w colluders must have at least $w(2 \lg n-\lg w-1)$ keys.

Proof. Let $A, B$ be any two communicating sets, and let $C_{1}, C_{2}, \ldots, C_{w}$ be any possible $w$ colluding sets. Then we must have

$$
A \cap B \nsupseteq C_{1} \cup C_{2} \cup \cdots \cup C_{w} .
$$

Suppose we take all

$$
M=\left(\begin{array}{l}
n \\
2
\end{array}\right)
$$

possible intersections pairs of sets $S_{i} \cap S_{j}$. Then all $\left(\begin{array}{l}M \\ w\end{array}\right)$ unions of these intersections must form a set antichain. Otherwise for some sets

$$
\left(A_{1} \cap B_{1}\right) \cup \cdots \cup\left(A_{w} \cap B_{w}\right) \subseteq\left(C_{1} \cap D_{1}\right) \cup \cdots \cup\left(C_{w} \cap D_{w}\right) .
$$

However, this contradicts (4). Note that some of the pairs on the left could be the same as some on the right, and there is no contradiction for such pairs. Nevertheless, for some $i$, the pair $A_{i}, B_{i}$ is not one of the pairs on the right side. For this pair we may select, from each pair $C_{j}, D_{j}$, one which is neither $A_{i}$ nor 
$B_{i}$. This set of choices allows us to construct the contradiction of (4) required. Thus we must have

$$
\left(\begin{array}{c}
k \\
\lfloor k / 2\rfloor
\end{array}\right) \geq\left(\begin{array}{c}
M \\
w
\end{array}\right)
$$

The bound follows from this by easy manipulations.

We note that set families in which no set is contained in the union of $w$ others have been studied in much greater depth is Erdős et al. [6]. Nevertheless even from our simple argument we can see that we must have $k$ growing at least linearly with the number of potential colluders. However, it seems difficult to achieve this. By a randomized construction we can achieve:

Theorem 4. A set intersection scheme exists which is secure against $w$ colluders and has $\left[\left((w+2)^{w+3} / 4 w^{w}\right) \ln n\right\rceil$ keys.

Proof. Suppose we choose the sets randomly as before with probabilities $p$. In the notation of the proof of Theorem 3, the probability of a successful collusion by fixed sets $C_{1}, \ldots, C_{w}$ against $A, B$ is

$$
\operatorname{Pr}\left(A \cap B \subseteq C_{1} \cup \cdots \cup C_{w}\right)=\left(1-p^{2}(1-p)^{w}\right)^{k} .
$$

This is minimized by putting $p=2 /(w+2)$, giving

$$
\begin{aligned}
\operatorname{Pr}\left(A \cap B \subseteq C_{1} \cup \cdots \cup C_{w^{\prime}}\right) & =\left(1-\frac{4 w^{w^{w}}}{(w+2)^{w+2}}\right)^{k} \\
& \leq \exp \left(-\frac{4 k w^{w}}{(w+2)^{w^{w}+2}}\right) .
\end{aligned}
$$

Thus the expected number of successful collusions is less than

$$
\frac{1}{2 w !} n^{w+2} \exp \left(\frac{-4 k w^{w}}{(w+2)^{w+2}}\right) \leq \frac{1}{2 w !}
$$

if $k \geq\left((w+2)^{w+3} / 4 w^{w}\right) \ln n$.

Corollary 1. A set intersection scheme exists which is secure against $w$ colluders and has less than $\left\lceil 2(w+2)^{3} \ln n\right\rceil$ keys.

Proof.

$$
\frac{(w+2)^{w+3}}{4 w^{w^{\prime}}}<\frac{e^{2}(w+2)^{3}}{4}<2(w+2)^{3} .
$$

The method of Theorem 4 can be derandomized as in Section 4 .

From Theorem 4 we can obtain a scheme guaranteed secure against any five colluders, in a network of 5000 users, with 3928 keys. Each user would have 
about 1123 keys on average. To be guaranteed secure for large $n$ against five colluders, the multiline scheme of [7] requires $n$ keys, with each user holding at least $16 \sqrt{n}$ keys. For $n=5000$ this would be 5000 keys in total, with each user holding at least 1131 . The multimap scheme of [7] only requires each user to have $12 \sqrt{n}$ keys but requires $6 n$ keys in total. For $n=5000$ this gives 30,000 keys with each user having at least 848 . For $n=10,000$ our scheme and the multimap scheme both require about 1200 keys per user, but our scheme needs just 4248 keys against 60,000 keys for multimap. For $n=100,000$ the corresponding figures would be 5310 with each user holding an average of 1517 keys for our scheme, 100,000 keys with each user holding at least 5059 for the multiline scheme, and 600,000 keys with each user holding at least 3794 for the multimap scheme.

There is one drawback of the randomized construction here (and of its derandomization). If we wish to.check that the scheme meets its security specification, the computational burden will grow like $\Omega\left(n^{w+2} k\right)$. This would clearly be infeasible for large $n$, if $w$ is not very small. From a practical point of view, the answer is to make the probabilities sufficiently small. However, we can show that, at the expense of more keys, we can construct schemes which are checkable (or derandomizable) in time little more than required by the basic scheme of Section 2.

Theorem 5. A set intersection scheme, secure against $w$ colluders, requiring less than $\left\lceil 500 w^{3} \ln n\right\rceil$ keys in total, exists which can be checked, or constructed deterministically, in $O\left(n^{3} k\right)$ time.

Proof. Choose $n$ sets from $k=500 w^{3} \ln n$ with probability $p=1 /(5 w)$. If $A, B$ are the communicating sets, it is sufficient to ensure that, for any set $C$,

$$
|C \cap A \cap B|<\frac{|A \cap B|}{w} .
$$

Clearly, this implies (4) for any collection $C_{1}, \ldots, C_{w}(i=1, \ldots, w)$. We bound the probability of (5) as follows, using a version of Chernoff's bound for the tails of the binomial distribution. (See, for example, [1].)

$$
\begin{aligned}
\operatorname{Pr}\left(|A \cap B| \leq 0.68 p^{2} k\right) & \leq \exp \left(-\frac{1}{2}(0.32)^{2}(0.2)^{2} 500 w \ln n\right) \\
& \leq n^{-2.048} \quad(w \geq 2) .
\end{aligned}
$$

Hence,

$$
\operatorname{Pr}(\text { any such } A, B \text { exist }) \leq\left(\begin{array}{l}
n \\
2
\end{array}\right) n^{-2.048}<\frac{1}{2}
$$


Now, for fixed $C$,

$$
\begin{aligned}
\operatorname{Pr}\left(|A \cap B \cap C| \geq \frac{0.68 p^{2} k}{w}\right) & =\operatorname{Pr}\left(|A \cap B \cap C| \geq 3.4 p^{3} k\right) \\
& \leq\left(\frac{e}{3.4}\right)^{3.4 p^{3 k}} \\
& <n^{-3.04}
\end{aligned}
$$

(For a proof of (7) see, e.g., Theorem I.7(ii) of [4].)

Hence,

$$
\operatorname{Pr}(\text { any such } A, B, C \text { exist }) \leq n\left(\begin{array}{l}
n \\
2
\end{array}\right) n^{-3.04}<\frac{1}{2} .
$$

From (6) and (8) it follows that the scheme exists with nonzero probability. The checking or derandomization of the scheme involves establishing the conditions (5) for all triples. This can be achieved by a similar method to that discussed in Theorem 2.

More practical versions of the schemes suggested here could be developed, as in Section 3, but we do not do so here.

\section{Groups of Users}

Mitchell and Piper [8] also consider a generalization of the problem considered here, where every subset of users of size at most $g$, for some given $g$, needs to have a key known only to the members of the group. The key is constructed by using intersections of sets taken $g$ at a time, rather than in pairs as we have done so far. Let us call such schemes $g$-intersection schemes. (Thus a set intersection scheme is a 2 -intersection scheme.) Our methods readily generalize to this situation. Thus we may prove a lower bound analogous to Theorem 3 .

Theorem 6. Any g-intersection scheme which is secure against $w$ colluders must have $k \geq w(g \lg n-\lg w-g \lg g)$.

Proof. We use the same method as for Theorem 3, but consider all intersections of sets taken $g$ at a time, and let

$$
M=\left(\begin{array}{l}
n \\
g
\end{array}\right) \text {. }
$$

To avoid cumbersome notation, we introduce some temporary terminology. The word block means a set of keys assigned to one of the $n$ users, and a group an intersection of some $g$ blocks. We use the term collusion to mean a set of $w$ groups. Now the $\left(\begin{array}{c}M \\ w\end{array}\right)$ sets, formed by taking the unions of groups in each possible collusion, are an antichain. Otherwise, the union of some collusion is contained in some other. To show that this implies a contradiction to the 
security assumption, suppose the union of collusion $A$ is contained in that of collusion $B$. Now some group in $A$ clearly does not appear in $B$. Let the blocks forming this group be $C_{1}, \ldots, C_{g}$, the group being $\cap_{i=1}^{g} C_{i}$. Now, for each group in $B$, we can select a block which is not one of the $C_{i}$. The union of these $w$ blocks now contains the intersection of the $C_{i}$, giving the contradiction. Thus we have

$$
\left(\begin{array}{c}
k \\
\lfloor k / 2\rfloor
\end{array}\right) \geq\left(\begin{array}{l}
M \\
w
\end{array}\right)
$$

which implies $2^{k} \geq(M / w)^{w} \geq\left((n / g)^{g} / w\right)^{w}$, giving the bound.

Corresponding to Theorem 4 we have:

Theorem 7. Ag-intersection scheme exists which is secure against $w$ colluders and has $\left.\left[\left((w+g)^{w+g+1} / g^{g} w^{w}\right)\right) \ln n\right\rceil$ keys.

Proof. The proof is similar to that of Theorem 4 . Selecting the sets randomly with probability $p$, now the probability of successful collusion is

$$
\operatorname{Pr}\left(A_{1} \cap \cdots \cap A_{g} \subseteq C_{1} \cap \cdots \cap C_{w}\right)=\left(1-p^{g}(1-p)^{w}\right)^{k} .
$$

This is minimized by putting $p=g /(w+g)$. Then

$$
\begin{aligned}
\left(1-p^{g}(1-p)^{w}\right)^{k} & =\left(1-\frac{g^{g} w^{w}}{(w+g)^{w+g}}\right)^{k} \\
& \leq \exp \left(-\frac{k g^{g} w^{w}}{(w+g)^{w+g}}\right) .
\end{aligned}
$$

The expected number of successful collusions is at most

$$
\frac{1}{g ! w !} n^{w+g} \exp \left(-\frac{k g^{g} w^{w}}{(w+g)^{w^{w}+g}}\right) \leq \frac{1}{g ! w !}
$$

if $k \geq\left((w+g)^{w+g+1} / g^{g} w^{w^{\prime}}\right) \ln n$.

Again we can derandomize the construction if required.

\section{Concluding Remarks}

The basic scheme we propose is within a small factor (i.e., less than 6.5 times) of optimal. In practice, using the method of Section 3, we can do even better than this. It would be exceedingly difficult to find deterministic combinatorial constructions having the required property which are as small. However, collusionproof schemes seem much harder to produce. Note that the order of growth in $w$ of the upper bound of Corollary 1 is $\Omega\left(w^{3}\right)$, as opposed to $\Omega(w)$ in the lower bound of Theorem 3. Establishing the correct order of growth remains an open 
question. This is a crucial question since the bound of Theorem 4 is rather large if $w$ is appreciable (and the bound of Theorem 5 is larger). When we consider groups, as in Section 6, the situation is even worse.

A possible criticism of our scheme, by comparison with those based on block designs [8], might be that the set intersections will vary in size. This is not really a practical difficulty, however, since if required we can always "pad out" all intersections to a standard length using "dummy" keys known to all users.

We have given methods for checking and derandomization, but these are clearly practicable only for relatively small $n$, unless massive parallelism is available. In practice, the schemes we propose would be generated randomly if $n$ is large, ensuring that the failure probabilities remain very small. This may raise questions about the practical production of large numbers of random bits. However, the same question could be raised for the myriad other successful applications of random numbers. It is usual practice to employ a suitably guaranteed pseudorandom source, and at present this seems the path one must follow. However, pseudorandomness seems to be an unnecessary device when (at least according to the quantum theory) nature itself is subject to continual and unavoidable random fluctuation. Randomized algorithms and constructions are becoming widely used, in addition to the already widespread use of random simulations. Consequently, there seems a clear need for the development of cheap physical devices for generating truly random bits, rather than cleverer ways of generating pseudorandom bits. As a modest example, such devices would allow the schemes proposed here to be generated simply and conveniently, and easily extended whenever desired.

\section{Acknowledgement}

The authors thank Dr. Y. Kohayakawa for helpful discussions.

\section{References}

[1] D. Angluin and L. G. Valiant, Fast probabilistic algorithms for Hamiltonian circuits and matchings, Journal of Computer and System Sciences 18 (1979), 155-193.

[2] R. Blom, Non-public key distribution, in Adiances in Cryptology: Proceedings of Eurocrypt 82, Plenum, New York, 1983, pp. 231-236.

[3] R. Blom, An optimal class of symmetric key generation systems, in Advances in Cryptology: Proceedings of Eurocrypt 84, Lecture Notes in Computer Science, Vol. 209, Springer-Verlag, Berlin, 1984, pp. 335-338.

[4] B. Bollobás, Random Graphs, Academic Press, London, 1985.

[5] P. Erdős, P. Frankl, and Z. Füredi, Families of finite sets in which no set is covered by the union of two others, Journal of Combinatorial Theory, Series A 33 (1982), 158-166.

[6] P. Erdős, P. Frankl, and Z. Füredi, Families of finite sets in which no set is covered by the union of $r$ others, Israel Journal of Mathematics 51 (1985), 79-89.

[7] Li Gong and D. H. Wheeler, A matrix key distribution scheme, Journal of Cryptology 2 (1990), 51-59.

[8] C. J. Mitchell and F. C. Piper, Key storage in secure networks, Discrete Applied Mathematics 21 (1988), 215-228.

[9] J. Spencer, Ten Lectures on the Probabilistic Method, SIAM, Philadelphia, PA, 1987. 